From the Cancer Genomics Program, Department of Oncology, University of Cambridge, Hutchison/MRC Research Centre, Cambridge, United Kingdom; and Division of Hematology/Oncology, Department of Medicine, University of North Carolina, Chapel Hill, NC.

Submitted July 6, 2005; accepted July 19, 2005

Terms in blue are defined in the glossary, found at the end of this issue and online at www.jco.org

Authors' disclosures of potential conflicts of interest are found at the end of this article.

Address reprint requests to Carlos Caldas, MD, Cancer Genomics Program Department of Oncology, University of Cambridge, Hutchison/MRC Research Centre, Cambridge, United Kingdom CB22XZ; e-mail: cc234@cam.ac.uk.

0732-183X/05/2329-7350/\$20.00 DOI: $10.1200 / J C O .2005 .03 .3845$

\title{
Molecular Classification and Molecular Forecasting of Breast Cancer: Ready for Clinical Application?
}

James D. Brenton, Lisa A. Carey, Ahmed Ashour Ahmed, and Carlos Caldas

$$
\begin{array}{llllllll}
\text { A } & \text { B } & \mathbf{S} & \mathbf{T} & \mathbf{R} & \mathbf{A} & \mathbf{C} & \mathbf{T}
\end{array}
$$

Profiling breast cancer with expression arrays has become common, and it has been suggested that the results from early studies will lead to understanding of the molecular differences between clinical cases and allow individualization of care. We critically review two main applications of expression profiling; studies unraveling novel breast cancer classifications and those that aim to identify novel markers for prediction of clinical outcome. Breast cancer may now be subclassified into luminal, basal, and HER2 subtypes with distinct differences in prognosis and response to therapy. However, profiling studies to identify predictive markers have suffered from methodologic problems that prevent general application of their results. Future work will need to reanalyze existing microarray data sets to identify more representative sets of candidate genes for use as prognostic signatures and will need to take into account the new knowledge of molecular subtypes of breast cancer when assessing predictive effects.

J Clin Oncol 23:7350-7360.

\section{INTRODUCTION}

The management of breast cancer has been dramatically changed with the advent of widespread screening programs and the systematic use of adjuvant hormonal therapy and chemotherapy. Recent data have shown that these changes are having a major impact in outcome, and despite increasing incidence, breast cancer mortality is decreasing in most of the Western world. ${ }^{1}$ The recent overview of randomized adjuvant therapy trials has confirmed that systemic therapies (hormone therapy and chemotherapy) are producing cures; survival curves at 15 years show a greater separation, despite recurrence curves' remaining almost flat after 5 years. ${ }^{2}$ Moreover the overview also confirms that tamoxifen is of benefit in only patients with estrogen receptor (ER) -positive disease, effectively representing a form of targeted therapy. Great excitement has also come from recent preliminary reports that appear to show the great benefit of trastuzumab therapy either concurrent or sequential with adjuvant chemotherapy for patients with HER2-positive tumors. These examples give further credence to the old idea that breast cancers are an heterogeneous group of diseases. This has been further confirmed by molecular profiling of breast cancers using array technology showing the biologic and clinical heterogeneity of breast cancer is explained by differences in the genetic composition of the primary tumors.

The questions that face clinicians treating breast cancer patients have remained despite all this progress: how to identify patients with node-negative disease at very low risk of relapse, and hence for whom the risk-benefit ratio might be in favor of withholding chemotherapy; how to predict the response to currently available treatments, in particular cytotoxic chemotherapy; and finally, the identification of tumor targets for directed therapies. The promise is that expression profiling of tumor RNA (or other profiling technologies, eg, arraybased comparative genomic hybridization for tumor DNA copy number profiling) will have practical application by helping make these difficult decisions more precise. ${ }^{3}$ 
Here we will critically review two types of molecular profiling studies using expression array technology, those that have unraveled a novel molecular taxonomy of breast cancers and those that have attempted to use gene expression analysis to predict clinical outcomes.

\section{MOLECULAR CLASSIFICATION OF BREAST CANCER}

The explosion of genomics technology has resulted in a wave of efforts to use these advances to improve patient care. This is nowhere more obvious than in breast cancer, where our inability to accurately predict relapse or response to therapy has resulted in a long history of single-marker prognostic and predictive studies. Those studies were hampered by the usual problems in marker trials such as too-small sample size, heterogeneous patients, tumors, and treatments, and lack of independent validation. Added to these problems in translational trial design was the fact that clinician-researchers had little understanding of how these markers related to one another and little means to decide which of several promising markers might be the most relevant. Expression profiling and other "-omic" technologies may help us begin to leap this hurdle. Since multiple genes are examined simultaneously without the requirement for a priori definition of relevant patterns, profiling studies can be performed even when we are somewhat ignorant of the relationships or the function of the genes examined. The results of these studies can inform both the clinician and the scientist. In this way, the usual sequence of scientific discovery followed by clinical application is streamlined, producing both clinical and scientific knowledge in parallel. In breast cancer, this is giving us a rapidly evolving understanding of the pathways responsible for outcome and response to therapy, and has also provided a wealth of new knowledge of targetable differences in breast cancer that will be the means to better therapy in the future.

As mentioned above, the clinical impression of breast cancer heterogeneity has been proven at the gene expression level. ${ }^{4}$ Using cDNA microarrays and unsupervised (without knowledge of clinical end points) analysis, investigators determined that there were breast cancer subtypes with distinct gene expression patterns (Fig 1) and different prognoses $^{5-6}$ (Fig 2) that persisted in primary breast cancers as well as their metastases. ${ }^{7}$ These groups of tumors confirmed long-recognized clinical differences in phenotype, but added new knowledge regarding breast cancer biology. For example, the gene expression profiling revealed that within the ER-related tumors at least two subtypes, luminal A and luminal B, could be distinguished that vary markedly in gene expression and prognosis. ${ }^{5}$ Conversely, hormone receptor-negative breast cancer comprised two distinct subtypes, the HER2 subtype and the basal-like subtype. ${ }^{5,8}$ These subtypes differ in biology and behavior, and both show a poor outcome. Importantly a very similar classification of breast cancers has now been characterized using immunohistochemistry to analyze patterns of protein expression in tumor sections and suggesting that a few protein biomarkers (eg, ER, progesterone receptor [PR], HER2, HER1, basal cytokeratins) can be used to stratify breast cancers into different groups that can be mapped to the subtypes outlined in the following sections. ${ }^{9-11,32}$

\section{LUMINAL SUBTYPES}

\section{Expression Patterns}

The luminal subtypes make up the hormone receptorexpressing breast cancers, and have expression patterns reminiscent of the luminal epithelial component of the breast. ${ }^{4}$ These patterns include expression of luminal cytokeratins 8/18, ER and genes associated with ER activation such as LIV1 and CCND1 (also known as cyclin D1). ${ }^{4,6}$ Fewer than $20 \%$ of luminal tumors have mutations in TP53, and these tumors are often grade I. ${ }^{5,6}$ Within the luminal cluster there are at least two subtypes, luminal A and luminal B. Although both are hormone receptor expressing, these two luminal subtypes have distinguishing characteristics. Luminal A has, in general, higher expression of ER-related genes and lower expression of proliferative genes than luminal $\mathrm{B} .{ }^{5,8}$

\section{Clinical Features}

Luminal breast cancers are the most common subtype of breast cancer. In a population-based case-control study, the Carolina Breast Cancer Study, luminal breast cancers represented $67 \%$ of the tumors. ${ }^{12}$ The incidence of the best-prognosis luminal A subtype differed by race and age in that premenopausal African American women who developed breast cancer were significantly less likely to develop luminal A tumors (36\%) than any other group of patients (51\% to 59\%). As discussed in the next section, this decreased incidence of the good-prognosis luminal A tumors among young African Americans was associated with an increase in the poor-prognosis basal-like breast cancer subtype. In addition, the luminal B tumors tend to be higher grade than luminal A tumors.

\section{Treatment Response and Outcome}

In general, the luminal subtypes carry a good prognosis; however there is clearly a difference in outcome, with luminal B having a significantly worse prognosis than luminal A in multiple data sets. ${ }^{8}$ Part of this different outcome may be due to variations in response to treatment. Luminal breast cancers are treated with hormone therapy. Several studies have demonstrated that ER-positive tumors respond poorly to conventional chemotherapy. ${ }^{13,14}$ It is likely however, that response may differ between luminal subtypes. A recent evaluation and validation of a 


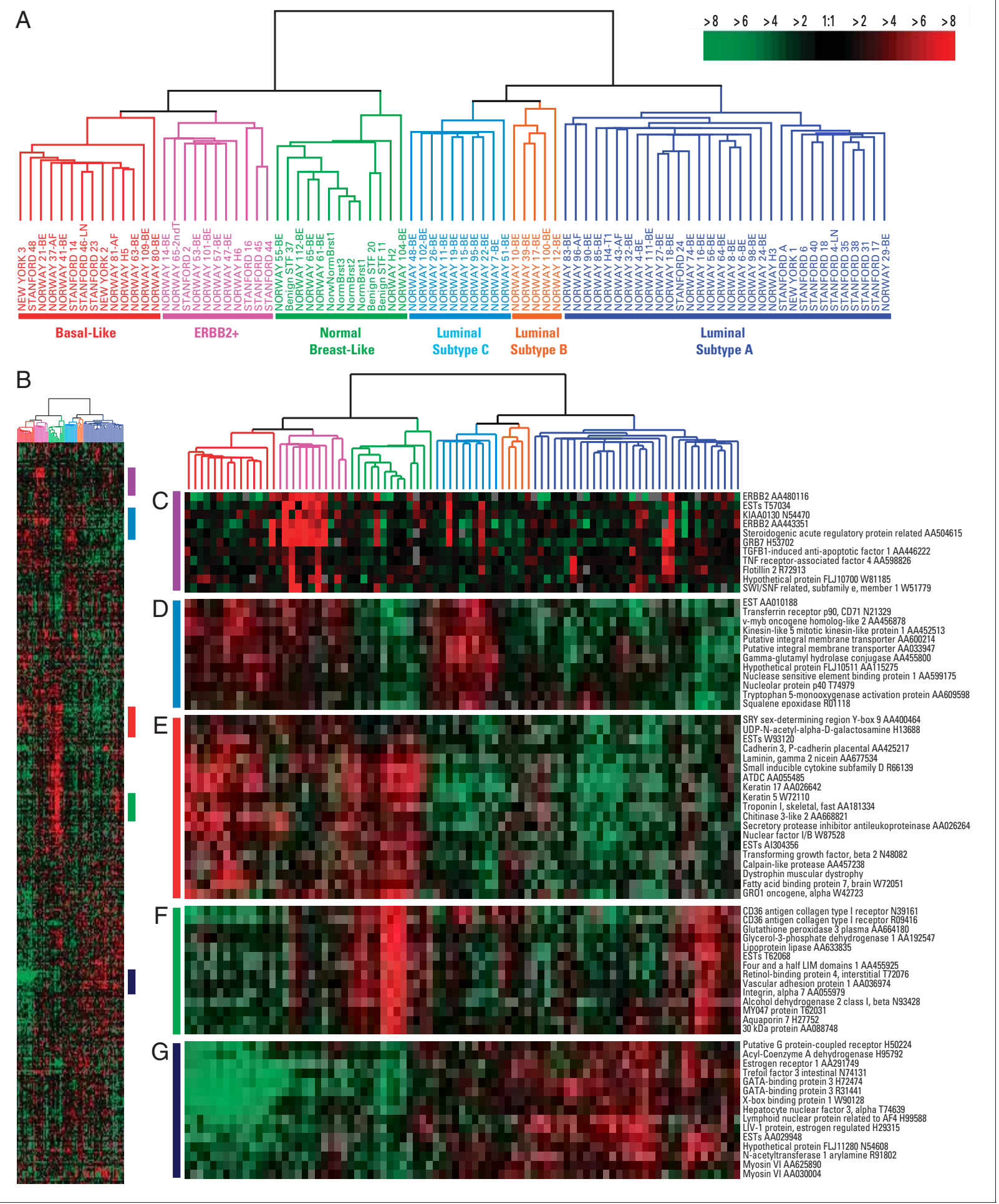

Fig 1. Gene expression patterns of 85 samples (78 carcinomas, three benign tumors, four normal tissues) analyzed by hierarchical clustering using the 476 cDNA intrinsic clone set. (A) The tumor specimens were divided into subtypes based on differences in gene expression. The cluster dendrogram showing the subtypes of tumors are colored as: luminal subtype A, dark blue; luminal subtype B, yellow; luminal subtype C, light blue; normal breast-like, green; basal-like, red; and ERBB2+, pink. (B) The full cluster diagram scaled down. The colored bars on the right represent the inserts presented in C-G. (C) ERBB2 amplicon cluster. (D) Novel unknown cluster. (E) Basal epithelial cell-enriched cluster. (F) Healthy breast-like cluster. (G) Luminal epithelial gene cluster containing ER. Reprinted with permission from Sorlie et al. ${ }^{5}$ 

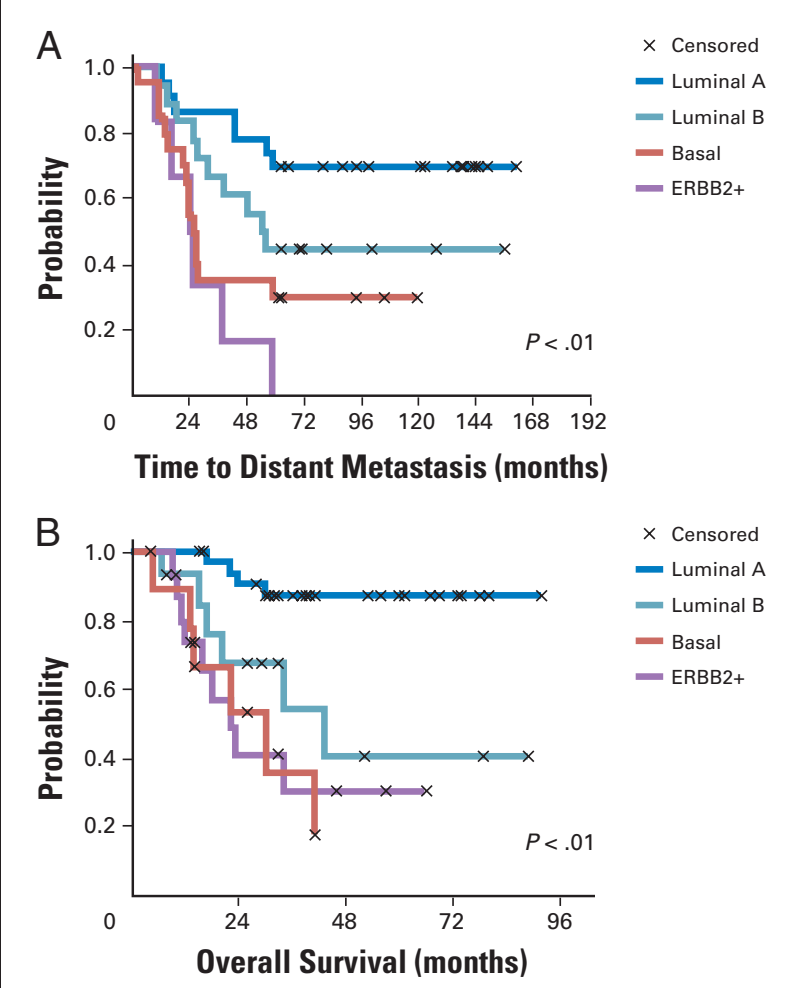

Fig 2. Disease outcome in independent patient cohorts. (A) Time to development of distant metastasis in the 97 sporadic cases from van't Veer et al (B) Overall survival for 72 patients with locally advanced breast cancer in the Norway cohort. Reprinted with permission from Sorlie et al. ${ }^{8}$

reverse transcriptase polymerase chain reaction-based 16-gene predictor of distant relapse among hormone receptor-positive node-negative patients treated with tamoxifen resulted in the Recurrence Score. ${ }^{15}$ The Recurrence Score has been shown to be prognostic in untreated patients, ${ }^{16}$ predictive of lack of efficacy of tamoxifen ${ }^{16}$ and predictive of response to chemotherapy. ${ }^{17,18}$ Eight of the 16 genes included in the Recurrence Score, including ER-related genes and proliferative genes, are included in the gene sets distinguishing luminal A from B (D. Oh, manuscript submitted for publication), and the data suggest that tumors with low Recurrence Scores are luminal A whereas those with high Recurrence Scores are luminal B. Thus, it appears that luminal A tumors may be quite adequately treated with endocrine therapy alone, whereas the more proliferative luminal B tumors may be those that benefit from chemotherapy added to endocrine therapy. Another pressing question is the response to tamoxifen therapy in luminal B patients since the scant data so far suggest that in the presence of tamoxifen, luminal $\mathrm{B}$ patients do significantly worse than luminal $A^{5,8}$; it may be that luminal $B$ patients will do better if they receive an aromatase inhibitor, or it may be that they are entirely endocrine therapy refractory. Future studies will be required to address this question.
There is a suggestion that other targeted approaches beyond endocrine therapy may work in luminal subtypes. The anti-vascular endothelial growth factor antibody bevacizumab was recently shown to improve survival in metastatic breast cancer when combined with paclitaxel. ${ }^{19}$ Intriguingly, more than $60 \%$ of the patients in that trial were hormone receptor-positive and virtually none were HER2-positive, suggesting that antiangiogenic strategies may be effective in the luminal subtypes.

\section{HER2 SUBTYPE}

\section{Expression Patterns}

The identification of a HER2-array subtype within unsupervised cluster analysis of breast cancers was reassuring to clinicians because it confirmed the clinical impression that tumors with HER2 overexpression were systematically different from other breast cancers. The array designation of HER2 (hereafter designated HER2array) should not be confused with HER2-positive tumors identified by immunohistochemistry or fluorescence in situ hybridization (FISH), because not all clinically HER2-positive tumors show the RNA expression changes that define the cluster. The HER2-array subtype refers to the larger group of hormone receptor-negative (low expression of ER and related genes by array) tumors identified by gene expression array. HER2-positive tumors clinically refer to those identified by immunostaining for HER2 overexpression or FISH for excess gene copy number. Most tumors that are clinically HER2-positive will fall within the HER2-array subtype. However, other tumors that are HER2 positive by immunohistochemistry or FISH will also overexpress hormone receptors, and most of these tumors fall within the luminal subtypes rather than the HER2-array subtype. ${ }^{5,6,8}$ In this section, we are referring only to the HER2-array subtype, which does not express hormone receptors. The HER2-array tumors are characterized by overexpression of other genes in the ERBB2 amplicon such as GRB7.4 Like basal-like tumors, the HER2-array subtype tumors have a high proportion, $40 \%$ to $80 \%$, of TP53 mutations, ${ }^{5,12}$ and are significantly more likely to be grade $3(P=.0002)$ than luminal A tumors.

\section{Clinical Features}

There is no association of the HER2-array subtype with either age or race, ${ }^{12}$ and no association with known risk factors. An intriguing recent analysis from the Nurses' Health Study suggests that hormonal risk factors do not predict ER-negative breast cancer, ${ }^{20}$ suggesting that investigators in large epidemiologic studies may do well to revisit the analyses of traditional risk factors in a subtype-specific manner. Since both HER2-array and basal-like breast cancers fit in the ER-negative category, 
it remains to be seen whether these risk factors also differ between the two main categories of ER-negative tumors. In addition to being more likely to be high grade and poorly differentiated, HER2-array subtype tumors were more than two-fold more likely to involve axillary lymph nodes than luminal A tumors. ${ }^{12}$

\section{Treatment Response and Outcome}

HER2-array subtype breast cancers carry a poor prognosis, ${ }^{5,6,8}$ another finding not surprising to those working with these patients and previously demonstrated in multiple single-marker studies. Despite its poor prognosis, the HER2-array subtype has also demonstrated sensitivity to anthracycline and taxane-based neoadjuvant chemotherapy, with significantly higher pathologic complete response than luminal breast tumors $(46 \% v 7 \% ; P<.001)$ in an M.D. Anderson Cancer Center (Houston, TX) study. ${ }^{13}$ Like the other ER-negative subtype, the basallike, the HER2-array subtype's poor prognosis seems to derive from a higher risk of early relapse among those without complete eradication of tumor cells. ${ }^{14}$ This finding is in keeping with a recent retrospective examination of Cancer and Leukemia Group B (CALGB) -led chemotherapy trials. In that study, the benefits of each generation of increasing chemotherapy aggressiveness-increased anthracycline dose and intensity (CALGB 8541), taxane added to anthracycline (CALGB 9344), and increased dose density (CALGB 9741) - were largely confined to the ER-negative subset of patients. ${ }^{21}$ This suggests that the HER2 and basal-like subtypes that make up the majority of ER-negative tumors are the tumors that derive the most benefit from improvements in chemotherapy. Unlike the basal-like, however, the HER2-array subtype has molecularly targeted agents, including the anti-HER2 monoclonal antibody trastuzumab. The effectiveness of trastuzumab in metastatic breast cancer as well as the remarkable reduction in relapse among HER2-positive breast cancers treated with trastuzumab combined with chemotherapy supports the idea that effective tumor control can be achieved with isolated targeting of this pathway $^{22-24}$ but not all HER2-positive tumors respond to trastuzumab. PTEN loss or abrogation, ${ }^{25}$ and CXCR4 upregulation $^{26}$ have been implicated in trastuzumab resistance and may provide targets for combination strategies for even better approaches in the future.

\section{BASAL-LIKE BREAST CANCER}

\section{Expression Patterns}

The basal-like subtype of breast cancer was so named because the expression pattern of this subtype mimicked that of the basal epithelial cells of other parts of the body and normal breast myoepithelial cells. ${ }^{4}$ These similarities include lack of expression of ER and related gene; low expression of HER2; strong expression of basal cytokeratins 5, 6, and 17; and expression of proliferationrelated genes. ${ }^{4,6}$ Immunohistochemical profiling using tissue microarray has identified that a group of tumors characterized by basal cytokeratin expression are also characterized by low expression of BRCA1. ${ }^{27}$ Indeed a basal phenotype is one of the hallmark features of "BRCA-ness" (sporadic cancers that look like those from $B R C A 1$ or BRCA2 mutation carriers) and might have important implications for management. ${ }^{28}$ Basal-like tumors are more likely to have aggressive features such as TP53 mutations and a markedly higher likelihood of being grade III $(P<.0001)$ than luminal A breast cancers $(P<.0001){ }^{5,12}$

\section{Clinical Features}

Some risk factors for developing basal-like breast cancers have been identified. As predicted by the hormone receptor and HER2-negative nature of these tumors, most women with BRCA1 mutations generally develop basal-like breast cancer. ${ }^{8,28}$ In the Carolina Breast Cancer Study, 20\% of the tumors were basal-like. However the basal-like subtype was most common among premenopausal African American women (39\%) compared with postmenopausal African American (14\%) or non-African American women of any age $(16 \%)(P=.0001) .{ }^{12}$ Interestingly, a recent study of risk among different racial and ethnic subtypes in the Women's Health Initiative suggested that among African American women, traditional risk factors such as menstrual and pregnancy history, body mass index, and activity failed to explain differences in incidence from white women. ${ }^{29}$ With the racial difference seen in the prevalence of cancer subtypes, this may mean that we need to re-evaluate the way we assess risk among different types of cancer.

\section{Treatment Response and Outcome}

Multiple independent data sets have revealed that the basal-like subtype carries a poor prognosis. ${ }^{5,6,8}$ Because premenopausal African American women have twice the risk of developing basal-like breast cancer as any other group of women, part of the poor prognosis experienced by these young African American women may be due to the subtype of breast cancer they develop. It is not clear if the poor prognosis of basal-like breast cancer is due to poor therapy options or inherent aggressiveness. Given its triple-negative receptor status (ER, PR, and HER2), basal-like breast cancer is not amenable to conventional targeted therapies for breast cancer such as endocrine therapy or trastuzumab, leaving only chemotherapy in the therapeutic armamentarium. Despite their poor prognosis, basal-like breast cancers are sensitive to conventional chemotherapy. Investigators at M.D. Anderson and at the University of North Carolina independently examined chemosensitivity in basal-like breast cancers. ${ }^{13,14}$ In both studies, response to anthracycline-based or combination anthracycline and taxane-based neoadjuvant 
chemotherapy was higher among basal-like breast cancer than non-basal like. These studies suggest that the poor prognosis experienced by patients with basal-like tumors is not from initial chemoresistance, but rather reflects the fewer treatment options available for ER-, PR-, and HER2-negative tumors and/or the intrinsic biology of this subtype.

This sensitivity to drugs may not be true of all agents. As mentioned, $B R C A 1$ pathway activity may be impaired in many basal-like breast cancers. BRCA1 functions in DNA repair and cell cycle checkpoint responses may result in sensitivity to DNA damaging agents, and resistance to spindle poisons. ${ }^{30}$ Regarding targeted options in this subtype, although none have yet been clinically proven, some preclinical studies suggest that this subtype may be epidermal growth factor receptor-driven. ${ }^{31,32}$ This hypothesis is being tested in several clinical trials. A recent publication examining the "wound response" signature, which includes genes involved in matrix remodeling and angiogenesis, found an association of this signature with basal-like subtype, suggesting other potential avenues of targeting. ${ }^{33}$

\section{MOLECULAR FORECASTING: PREDICTING OUTCOMES} WITH EXPRESSION PROFILING

Prognostic studies analyze variables that may predict future events and in most clinical studies these are related to time to death or recurrence. The main application of the new marker is to stratify patients by outcome and therefore allow better counseling and treatment decisions. The study of univariate prognostic factors in clinical cohorts is difficult and prone to multiple sources of bias. ${ }^{34}$ In contrast to prospective randomized trials, most prognostic studies are poorly designed and carried out in the absence of a predetermined written protocol, eligibility criteria, a primary end point or predefined statistical analysis. ${ }^{35}$ Prognostic variables should be tested in a representative sample of the population at risk at a common point in the course of their disease. ${ }^{36}$ The use of "available" samples does not satisfy this requirement and often results in inadequate clinical information for important clinical covariates, such as performance status and treatment. Consequently many studies provide data-driven analyses and report uncertain effects particularly in patient subgroups. Testing multiple variables from microarray or other high-throughput experiments adds further methodologic and statistical challenges to the conduct of prognostic studies.

Unsupervised methods such as cluster analysis (as described in the previous section) identify new partitions in data sets by comparing pair-wise similarity measures of gene expression. However these methods are subjective because different partitions can be identified with different mathematical methods of measuring similarities. Because these methods do not derive statistically valid information regarding which genes are differentially expressed between classes, they are poorly suited for identifying prognostic variables. ${ }^{37}$

The aim of supervised classification of data is to detect genes that may predict defined outcomes. The identification of a predictive multivariate marker from microarray expression data involves three main steps. ${ }^{35}$ First, invariant genes must be filtered from the data set to prevent noise obscuring true biologic associations. Then the remaining genes of interest are ranked by their strength of association with the outcome. Second, a model is identified which can predict outcome using the gene expression values as input to a mathematical formula. Third, a prediction rule is defined that categorizes the output from the model into clinically defined classes using cut-off points.

Internal validation is used as part of the model-building process to estimate the accuracy of the predictor in an unbiased manner. This requires a training set and a separate test set that should not be used to develop the model. Ideally, the test set should resemble the population from which future samples will be tested. Split-sample internal validation simply divides the samples into two sets. However, more efficient methods such as cross validation are commonly used. These methods test the model-building process, not the list of genes identified. Testing is performed as an iterative process by selecting different training and test sets from the series. In leave-one-out cross validation, test sets of one sample are chosen and the accuracy of the model derived from the remaining $(n-1)$ samples is scored. This is repeated for all $n$ samples so that every sample acts as a test set. The cross-validated predictive error for the model is obtained and can be used to compare different models or optimize the number of genes included in the model. All samples are then used to derive the final model. Although internal validation is an essential part of model development and refinement, it is not a replacement for formal external validation.

Several important problems arise using these methods. First, it is inevitable that for many hypothesis-generating microarray studies, the huge disproportion between the number of variables tested (gene expression values) and the number of samples leads to a high false-discovery rate for genes that appear to be associated with outcome. This can be controlled by using additional statistical tests (eg, the $\mathrm{q}$ value ${ }^{38}$ ), and the likelihood of selecting a particular list of genes by chance can be quantified using permutation tests (Fig 3). The predictive model generated is likely to reflect much of the biases in the training set rather than being a true representation of gene expression in the population of interest. The prediction model is therefore "overfitted" to the cohort under study, and a high predictive accuracy in the training set will not be reproducible in 


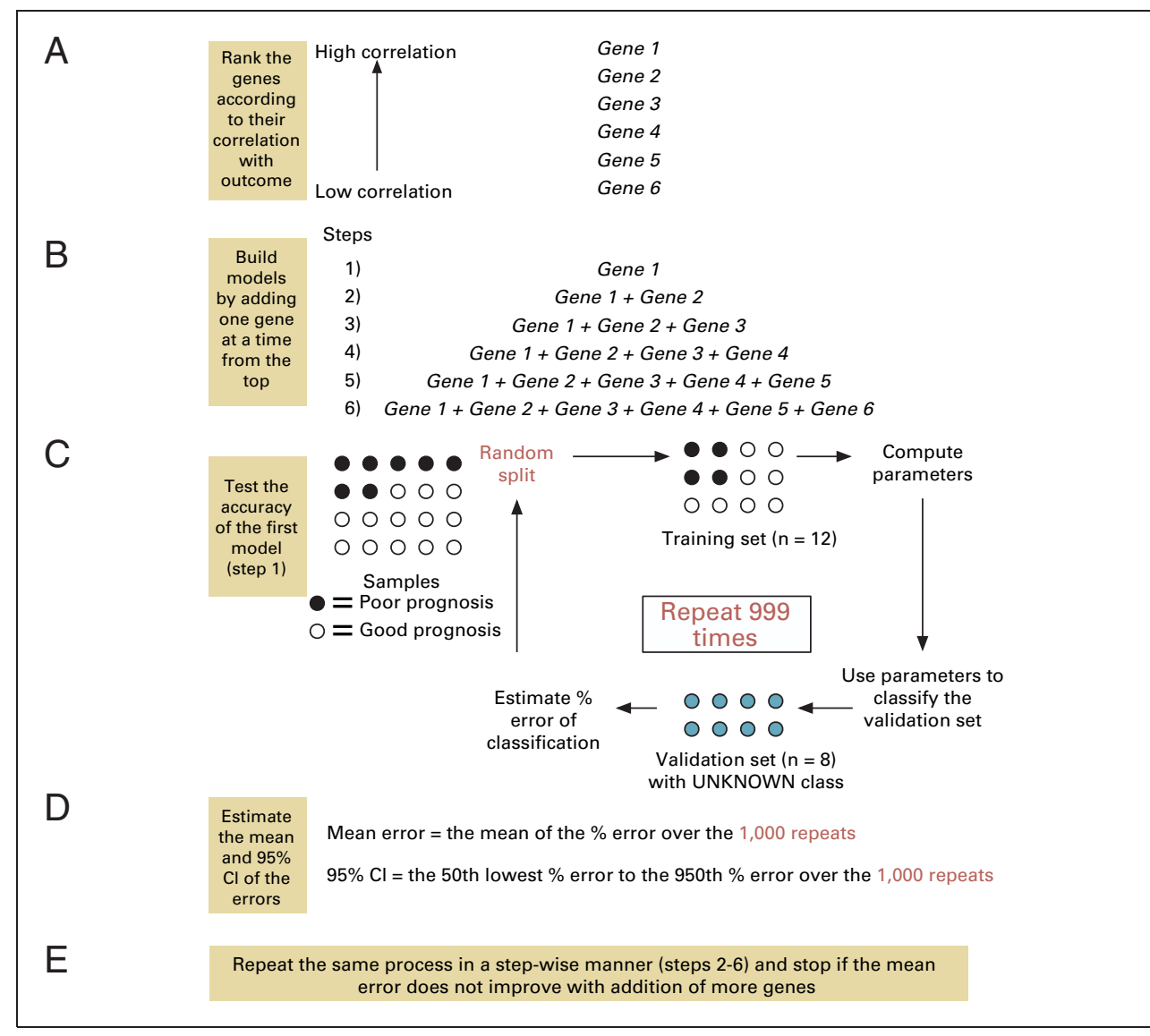

Fig 3. Cross-validation procedures in model building and estimation of the prediction error by permutation.

real samples. Incorrect internal validation, for example by performing resubstitution, not cross validation, dramatically underestimates the error rate by increasing over fitting. ${ }^{35,39}$ Other obvious biases, such as using external validation sets that are not independent of the training sets, make results very hard to interpret. Finally, the performance of the model is highly dependent upon the cutoff point used to classify outcomes. Arbitrarily defined cutoff values do not allow true estimation of the predictive power of the classifier and may be highly biased by the characteristics of the training set. Receiver operating characteristic (ROC) curves plot the sensitivity and specificity obtained for multiple cutoff points and allow objective assessment of the optimum value. Classifiers that have the largest area under the curve (AUC; shift of the curve to the left) have the best performance and AUC may be used to compare performance between different predictor models.

Many expression microarray studies have addressed broad prognostic questions in breast cancer ${ }^{5,6,40-45}$ but none satisfy previously defined criteria for good prognostic studies or provide a sufficient level of evidence for candidate markers (Table 1). The two largest studies have sought to identify markers for recurrence or survival after surgical treatment of node-negative disease. Van't Veer et $\mathrm{al}^{40}$ identified a cohort of 98 node-negative breast can- cers with onset before the age of 55 years. They compared expression patterns between those who relapsed within 5 years to those who were disease free at 5 years or more. Supervised methods identified a 70-gene signature, which was then applied to an additional set of 197 unique breast cancers. ${ }^{41}$ Combining both the original cancers and the follow-up set provided incomplete validation of the poor-prognosis signature and Cox proportional hazards analysis showed the signature to have a hazard ratio of 4.6 (95\% CI, 2.3 to 9.2) which outperformed other standard univariate clinical parameters. In a similar sized study, Wang et $\mathrm{al}^{45}$ derived a 76 -gene prognostic signature from 115 node-negative cancers and validated their signature on a wholly independent series of 171 samples. The Wang signature had a hazard ratio of $5.55(95 \%$ CI, 2.46 to 12.5 ) and again appeared to outperform univariate tests.

Strikingly, despite similar clinical and statistical designs, the two independent signatures share only three genes. This may be partly caused by the use of different microarray platforms, which can lead to differences in data repeatability and gene discovery. ${ }^{46-48}$ In addition, the training sets for both studies differed in mean age (44 years $v 54$ years) but there were no other major clinical differences. However, a more important effect may have 


\begin{tabular}{|c|c|}
\hline Microarray technology & $\begin{array}{l}\text { Reporting of methods of RNA extraction, probe preparation, probe labeling and hybridization } \\
\text { Reporting of microarray platform used, method of image analysis and segmentation methods }\end{array}$ \\
\hline Clinical design issues & $\begin{array}{l}\text { Clearly defined research question } \\
\text { Clear definitions for inclusion criteria and sample selection } \\
\text { All patients recruited at the same point in the course of the disease } \\
\text { The use of clinically relevant outcome measures } \\
\text { Use of clear definitions to characterize established prognostic factors (confounding variables) including details of } \\
\quad \text { measurement methods } \\
\text { Full description of treatment subsequent to inclusion in the cohort }\end{array}$ \\
\hline Statistical analysis & $\begin{array}{l}\text { Quality measures reported including assessment of feature quality, array background, spatial defects, results of } \\
\text { positive and negative control features on an array and repeatability across technical replicates } \\
\text { Clear description of the normalization process including an assessment of the degree of normalization needed before } \\
\text { downstream analysis } \\
\text { Clear description of the filtering process used before downstream analysis } \\
\text { Cross validation of classifiers should be performed on subsets that were not included in developing such a classifier } \\
\text { Use of receiver operating characteristic curves to estimate the performance of a classifier } \\
\text { External validation on independent data sets derived from prospective trials }\end{array}$ \\
\hline Raw data reporting & $\begin{array}{l}\text { Reporting of raw microarray images and raw intensity files } \\
\text { Reporting of detailed statistical analysis }\end{array}$ \\
\hline
\end{tabular}

been caused by marked variability in the derived prognostic signatures. Reanalysis of the original van't Veer data has shown that the predictive signature is not unique and indeed the data set contains multiple signatures that all correlate well with survival. ${ }^{49}$ This paradox arises because the difference between gene-outcome correlations is small, making it difficult to identify the most predictive genes, and the process of choosing the genes is highly dependent upon the subset of patients used to develop the prediction model. Independent reanalysis of other microarray data sets has revealed very similar findings. ${ }^{50}$ This has led others to propose a different algorithm for outcome prediction that treats survival as a continuous variable and then considers for subsequent unsupervised clustering only the genes with a Cox score that exceed a defined threshold. ${ }^{51}$

It is clear that more data are needed to identify key determinants of clinical outcome in node-negative breast cancer. Further refinement will require analysis of larger independent series of patients, but this is time consuming and expensive. There may be benefits from studying mouse models of breast cancer metastasis. Kang et $\mathrm{al}^{52}$ used in vivo selection in mice to identify subclones of the breast cancer cell line MDA-MB-231, with greatly enhanced metastatic specificity to bone. By passaging clonal lines through several generations and using microarray profiling, they identified 102 genes most strongly correlated with bone metastasis. Many of the overexpressed genes encoded proteins with osteolytic and angiogenic functions, including interleukin 11 and connective tissue growth factor. Downregulated genes included extracellular matrix and adhesion molecules. In an independent study of 19 primary breast cancers grouped by their ability to metastasize to bone or lymph nodes, Woelfle et $\mathrm{al}^{53}$ also identified a bone metastasis signature with repression of extracellular matrix and adhesion proteins that was distinct from that of lymph node metastasis alone. The ex- pression patterns seen in highly metastatic variants of MDA-MB-231 were similar to the van't Veer 70-gene signature, suggesting that the mouse may be a powerful model to refine meaningful gene selection. ${ }^{54}$

To identify a prognostic marker for early breast cancer treated with tamoxifen, $\mathrm{Ma}$ et $\mathrm{al}^{55}$ profiled 60 patients and identified nine genes that were significantly correlated with outcome from laser capture-dissected tumors. This was used to develop a cutoff for classification based on the ratio of two of the genes. When tested in an independent series of 20 patients, the two-gene expression ratio correctly predicted outcome in 16 of 20 patients. External validation of the marker performed by another group using quantitative polymerase chain reaction in an independent series of 58 patients with ER-positive breast cancer failed to show any significant predictive value. ${ }^{56}$ Although there were differences in the two breast cancer cohorts, both were small, and an accompanying editorial highlights how a high false-positive rate for gene discovery and lack of adequate model development may have contributed to the differences. ${ }^{58}$

Taken together, these results underscore the difficulties of using limited numbers of samples together with a sensitive technology that may be poorly reproducible. An alternative to using fresh-frozen material is to perform expression profiling on paraffin-embedded tissue, which is possible using multiplex real-time reverse transcriptase polymerase chain reaction. Using paraffin material for expression profiling is of particular importance because the success of screening programs have decreased tumor size at diagnosis and material is available from randomized trials, allowing well-controlled hypothesis-testing studies to be carried out.

Paik et $\mathrm{al}^{16}$ identified 250 candidate genes as potential prognostic factors in breast cancer from several gene expression studies and published literature. Expression of these candidates was tested in 233 samples from two 
National Surgical Adjuvant Bowel and Breast (NSABP) trials in which subjects with ER-positive, node-negative breast cancer received tamoxifen. Successful polymerase chain reaction could be carried out in paraffin blocks that were more than a decade old. A Recurrence Score algorithm was derived that predicted low (recurrence rates of $10 \%$ or less), intermediate (10\% to $30 \%)$ and high (more than 30\%) risk which combined the expression of 21 genes including five controls. The genes included those induced by estrogen and involved in proliferation and cell cycle control. Having generated the gene model, the authors used 668 samples from NSABP B-14 to validate these results. Further information from NSABP suggests that the 21-gene signature is prognostic in untreated women in B-14 and may also be predictive of benefit from adjuvant chemotherapy in B-20. ${ }^{15}$ In contrast to current data from expression microarray experiments, these results appear compelling. However several key issues remain. Although large, the validation set from B-14 was based on available samples, so unexpected biases are still possible. Validation of the recurrence score will require a randomized phase III study, which is currently being planned (US Intergroup Program for the Assessment of Clinical Cancer Tests [PACCT] study). External validation also requires use of the test in different laboratories to show that the methods are reproducible and widely applicable. The use of RNA presents a continuing challenge and differences in quantitation of ERBB2 were seen between B-14 and B-20 samples. Translation of these findings into an immunohistochemical test would make it easier to directly assess scores in tumor tissue in routine clinical practice.

New prognostic markers are often compared with existing markers (such as grade or stage) by showing differential effects on survival using Kaplan-Meier plots. Most often these comparisons do not integrate all that is known about the patient and in particular do not exclude whether the new marker can perform better than combinations of existing markers. A more definitive assessment can be derived from multivariable analysis in which a hazard ratio (HR) indicates the individual contribution in comparison with other established markers. Some caution is needed in interpretation of these values as the significance tests for the HR indicates the probability that the HR is not zero, not how significant is the contribution of the marker to prognosis. Eden et $\mathrm{al}^{58}$ compared the van't Veer 70 -gene marker with a combination of conventional multivariable prognostic indices and the Nottingham Prognostic Index on the original van't Veer data set. Comparison of the two markers was made using ROC curves and Kaplan-Meier plots, but no difference in performance was distinguishable. Both methods could predict more accurately for ER-positive as compared with ER-negative breast cancer. A realistic aim for new prognostic markers perhaps should be to improve performance, not replace existing markers. More sophisticated tests that can quantify the additional utility of a marker are urgently required. ${ }^{59}$

Predictive studies are a specialized form of prognostic studies where the outcome of interest is response to treatment and the application of the test is to inform which patients are suitable for specific therapy. These constraints make the identification of predictive signatures more difficult than prognostic factors and require prospectively controlled clinical trials. Chang et $\mathrm{al}^{44}$ used a sequential cohort of 24 locally advanced breast cancers to identify resistant and sensitive tumors to neoadjuvant docetaxel. Supervised classification identified a 92-gene predictor that correlated with docetaxel response. However, response was defined retrospectively using median residual volume, not standard clinical criteria, and the usefulness of the predictor is yet to be validated. Similar studies have used pathologic complete response and magnetic resonance imaging to define response from taxane-based treatment. ${ }^{60,61}$ In a follow-up expression study of 13 surgical specimens from the Chang cohort, the authors found strikingly little differences in gene expression between residual tumor from resistant and sensitive cases. ${ }^{62}$ The authors suggest that this may indicate selection for a resistant subpopulation of cells, but other confounding factors including over-representation of normal tissue expression have not yet been excluded.

\section{CONCLUSION}

So what are we clinicians to make of the two types of studies reviewed in the previous sections? On one hand we have molecular classifications that appear to be robust and reproducible and relatively trivial to apply in the clinic. For example, with just three markers (ER, PR, HER2) it is possible to define four major breast cancer subclasses with major therapeutic implications: $E R+1$ PR+/HER2-, ER+/HER2+, ER-/HER2+ and ER-/ PR-/HER2-. On the other hand we have the predictive signatures that so far have ignored most of this information and lumped all patients together. Would predictive signatures perform equally well in all these subclasses? The answer is unknown because the studies performed to date have included all patients irrespective of HER2 status, and so the potential impact on HER2-positive patients is not quantifiable. The situation is similar for triplenegative (ER-/PR-/HER2-) patients. A recent editorial in the Journal of Clinical Oncology ${ }^{63}$ suggests that retrospective analyses, such as the recent overview by the Early Breast Cancer Trialists Collaborative Group, ${ }^{2}$ that were derived from unselected patient populations, have questionable value for patient cohorts defined by, for example, HER2 status. We suggest that a similar maxim should be applied to predictive classifiers, and, hence, that candidate predictive signatures will need to be re-derived from wellcharacterized patient cohorts defined by virtue of clinical 
and biologic variables. We therefore question the feasibility of using existing signatures for prospective randomization of patients in clinical trials. If the early results from adjuvant trials confirm the strong benefit for HER2-positive patients using adjuvant chemotherapy plus trastuzumab, would there be clinicians prepared to withhold adjuvant chemotherapy in a young patient with a node-negative, HER2-positive breast cancer and a "good-prognosis" signature? And likewise for a similar patient with a triple-negative cancer, but for whom chemotherapy might be the only systemic therapy available to reduce risk of relapse?

We propose a way forward: (1) data from existing predictive gene expression signatures should be mined using different algorithms (eg, the recently proposed semisupervised analysis shown to outperform the classifiers derived using totally supervised approaches ${ }^{51}$ ) to try to identify an overlapping consensus set of candidate predictive genes that could then be validated externally using polymerase chain reaction-based assays; (2) larger retrospective studies that include hundreds of samples from each of the major subtypes defined by nodal status and a small number of markers (ER/PR/HER2, and possibly basal cytokeratins and EGFR) should be conducted using legacy tumor banks to generate both a more definitive breast cancer taxonomy and also a further set of predictive signatures to be validated both prospectively or in samples from completed clinical trials; and (3) prospective systemictherapy clinical trials should be designed with predictive marker validation in mind, following rigorous design (Sargent et $\mathrm{al}^{64}$ ).

The most difficult issue to tackle is going to be validation of predictive markers for node-negative disease, where a randomization decision of receiving systemic therapy on the basis of assigning a patient to a goodprognosis group might mean that such therapy is denied for someone who could benefit from it. The statistical modeling that will help make such decisions easier will require rigorous evaluation of the predictive power of signatures, including truly independent external validation, and their independence from tried, tested, trusted, and validated methodology (eg, the Adjuvant! software package) ${ }^{65}$ We remain skeptical that the predictive expression signatures have passed these litmus tests. The adherence to guidelines for evaluating new prognostic (and predictive) factors proposed long ago ${ }^{66}$ should ensure that the field progresses along a smoother path. The promise of genomics deserves no less.

\section{Authors' Disclosures of Potential Conflicts of Interest}

The authors indicated no potential conflicts of interest.

\section{REFERENCES}

1. Peto $R$, Boreham J, Clarke M, et al: UK and USA breast cancer deaths down $25 \%$ in year 2000 at ages 20-69 years. Lancet 355:1822, 2000

2. Early Breast Cancer Trialists' Collaborative Group: Effects of chemotherapy and hormonal therapy for early breast cancer on recurrence and 15-year survival: An overview of the randomised trials. Lancet 365:1687-1717, 2005

3. Caldas C, Aparicio SA: The molecular outlook. Nature 415:484-485, 2002

4. Perou CM, Sorlie T, Eisen MB, et al: Molecular portraits of human breast tumours. Nature 406:747-752, 2000

5. Sorlie T, Perou CM, Tibshirani R, et al: Gene expression patterns of breast carcinomas distinguish tumor subclasses with clinical implications. Proc Natl Acad Sci U S A 98:1086910874, 2001

6. Sotiriou C, Neo SY, McShane LM, et al: Breast cancer classification and prognosis based on gene expression profiles from a populationbased study. Proc Natl Acad Sci U S A 100:10393-10398, 2003

7. Weigelt B, Glas AM, Wessels LF, et al: Gene expression profiles of primary breast tumors maintained in distant metastases. Proc Natl Acad Sci U S A 100:15901-15905, 2003

8. Sorlie T, Tibshirani R, Parker J, et al: Repeated observation of breast tumor subtypes in independent gene expression data sets. Proc Natl Acad Sci U S A 100:8418-8423, 2003
9. Callagy G, Cattaneo E, Daigo Y, et al: Molecular classification of breast carcinomas using tissue microarrays. Diagn Mol Pathol 12:27-34, 2003

10. Abd El-Rehim DM, Pinder SE, Paish CE, et al: Expression of luminal and basal cytokeratins in human breast carcinoma. $J$ Pathol 203:661-671, 2004

11. Jacquemier J, Ginestier C, Rougemont J, et al: Protein expression profiling identifies subclasses of breast cancer and predicts prognosis. Cancer Res 65:767-779, 2005

12. Carey LA, Perou CM, Dressler LG, et al: Race and the poor prognosis basal-like breast cancer (BBC) phenotype in the population-based Carolina Breast Cancer Study. J Clin Oncol, 2004 (suppl; abstr 9510)

13. Rouzier R, Anderson K, Hess KR, et al: Basal and luminal types of breast cancer defined by gene expression patterns respond differently to neoadjuvant chemotherapy. San Antonio Breast Cancer Symposium. San Antonio, TX, 2004 (abstr 1026)

14. Carey LA, Dees EC, Sawyer L, et al: The triple negative paradox: Primary tumor chemosensitivity of the basal-like breast cancer (BBC) phenotype. San Antonio Breast Cancer Symposium. San Antonio, TX, 2004 (abstr 1023)

15. Paik S, Shak S, Tang G, et al: A multigene assay to predict recurrence of tamoxifen-treated, node-negative breast cancer. $N$ Engl J Med 351:2817-2826, 2004

16. Paik S, Shak S, Tang G, et al: Expression of the 21 genes in the Recurrence Score assay and tamoxifen clinical benefit in the NSABP study B-14 of node negative, estrogen receptor positive breast cancer. J Clin Oncol 23:6s, 2005 (suppl; abstr 510)

17. Paik S, Shak S, Tang G, et al: Expression of the 21 genes in the Recurrence Score assay and prediction of clinical benefit from tamoxifen in NSABP study B-14 and chemotherapy in NSABP study B-20, San Antonio Breast Cancer Symposium. San Antonio, TX, 2004 (abstr 24)

18. Gianni L, Zambetti M, Clark K, et al: Gene expression profiles of paraffin-embedded core biopsy tissue predict response to chemotherapy in patients with locally advanced breast cancer. J Clin Oncol, 2004 (suppl; abstr 501)

19. Miller KD, Burstein HJ, Elias A, et al: Phase II study of SU11248, a multitargeted receptor tyrosine kinase inhibitor (TKI), in patients (pts) with previously treated metastatic breast cancer (MBC). J Clin Oncol, 2005 (suppl; abstr 563)

20. Colditz GA, Rosner BA, Chen WY, et al: Risk factors for breast cancer according to estrogen and progesterone receptor status. J Natl Cancer Inst 96:218-228, 2004

21. Berry D, Cirrincione $C$, Henderson IC, et al: Effects of improvements in chemotherapy on disease-free and overall survival of estrogen receptor negative, node positive breast cancer: 20 years experience of the CALGB and US Breast Intergroup, San Antonio Breast Cancer Symposium. San Antonio, TX, 2004 (abstr 29)

22. Romond E, Perez EA, Bryant J, et al: Doxorubicin and cyclophosphamide followed by 
paclitaxel with or without trastuzumab as adjuvant therapy for patients with HER-2 positive operable breast cancer. Presented at $41 \mathrm{st}$ Annual Meeting of the American Society of Clinical Oncology, Orlando, FL, May 13-17, 2005

23. Perez EA, Suman $V$, Davidson $N$, et al: NCCTG N9831 May 2005 Update. J Clin Oncol, 2005 Presented at 41st Annual Meeting of the American Society of Clinical Oncology, Orlando, $\mathrm{FL}$, May 13-17, 2005

24. Piccart-Gebhart M: First Results of the HERA trial: A randomized three-arm multi-center comparison of 1 year herceptin, 2 years herceptin, or no herceptin in women with HER-2 positive primary breast cancer who have completed adjuvant chemotherapy. J Clin Oncol, 2005 Presented at 41st Annual Meeting of the American Society of Clinical Oncology, Orlando, $\mathrm{FL}$, May 13-17, 2005

25. Nagata $Y$, Lan $\mathrm{KH}$, Zhou $X$, et al: PTEN activation contributes to tumor inhibition by trastuzumab, and loss of PTEN predicts trastuzumab resistance in patients. Cancer Cell 6:117127, 2004

26. Tripathy D, Hassan S, Verma U, et al: Phenotypic and proteomic alterations of acquired trastuzumab resistence. J Clin Oncol, 2005 (suppl; abstr 3121)

27. Abd El-Rehim DM, Ball G, Pinder SE, et al: High-throughput protein expression analysis using tissue microarray technology of a large well-characterised series identifies biologically distinct classes of breast cancer confirming recent cDNA expression analyses. Int $\mathrm{J}$ Cancer 116:340-350, 2005

28. Turner $N$, Tutt $A$, Ashworth A: Hallmarks of 'BRCAness' in sporadic cancers. Nat Rev Cancer 4:814-819, 2004

29. Chlebowski RT, Chen Z, Anderson GL, et al: Ethnicity and breast cancer: Factors influencing differences in incidence and outcome. J Natl Cancer Inst 97:439-448, 2005

30. Kennedy RD, Quinn JE, Mullan PB, et al: The role of BRCA1 in the cellular response to chemotherapy. J Natl Cancer Inst 96:1659-1668, 2004

31. Sartor C, Zhou H, Perou CM, et al: Basallike breast tumor-derived cell lines are growth inhibited and radiosensitized by epidermal growth factor receptor (EGFR) tyrosine kinase inhibitors. San Antonio Breast Cancer Symposium. San Antonio, TX, December 8-11, 2004

32. Nielsen TO, Hsu FD, Jensen $K$, et al: Immunohistochemical and clinical characterization of the basal-like subtype of invasive breast carcinoma. Clin Cancer Res 10:5367-5374, 2004

33. Chang HY, Nuyten DS, Sneddon JB, et al: Robustness, scalability, and integration of a wound-response gene expression signature in predicting breast cancer survival. Proc Nat Acad Sci U S A 102:3738-3743, 2005
34. Levine MN, Browman GP, Gent M, et al: When is a prognostic factor useful? A guide for the perplexed. J Clin Oncol 9:348-356, 1991

35. Simon R: Diagnostic and prognostic prediction using gene expression profiles in highdimensional microarray data. $\mathrm{Br} \mathrm{J}$ Cancer 89: 1599-1604, 2003

36. Altman DG: Systematic reviews of evaluations of prognostic variables. BMJ 323:224-228, 2001

37. Simon R, Radmacher MD, Dobbin $K$, et al: Pitfalls in the use of DNA microarray data for diagnostic and prognostic classification. J Nat Cancer Inst 95:14-18, 2003

38. Storey JD, Tibshirani R: Statistical significance for genomewide studies. Proc Natl Acad Sci U S A 100:9440-9445, 2003

39. Simon R: When is a genomic classifier ready for prime time? Nat Clin Pract Oncol 1:4-5, 2004

40. van 't Veer LJ, Dai H, van de Vijver MJ, et al: Gene expression profiling predicts clinical outcome of breast cancer. Nature 415:530-536, 2002

41. van de Vijver MJ, He YD, van't Veer LJ, et al: A gene-expression signature as a predictor of survival in breast cancer. $N$ Engl $J$ Med 347:1999-2009, 2002

42. Ahr A, Karn T, Solbach $C$, et al: Identification of high risk breast-cancer patients by gene expression profiling. Lancet 359:131-132, 2002

43. Huang $E$, Cheng $\mathrm{SH}$, Dressman $\mathrm{H}$, et al: Gene expression predictors of breast cancer outcomes. Lancet 361:1590-1596, 2003

44. Chang JC, Wooten EC, Tsimelzon A, et al: Gene expression profiling for the prediction of therapeutic response to docetaxel in patients with breast cancer. Lancet 362:362369, 2003

45. Wang $Y$, Klijn JG, Zhang $Y$, et al: Geneexpression profiles to predict distant metastasis of lymph-node-negative primary breast cancer. Lancet 365:671-679, 2005

46. Lee JK, Bussey KJ, Gwadry FG, et al: Comparing cDNA and oligonucleotide array data: Concordance of gene expression across platforms for the NCl-60 cancer cells. Genome Biol 4:R82, 2003

47. Zorn KK, Jazaeri AA, Awtrey CS, et al: Choice of normal ovarian control influences determination of differentially expressed genes in ovarian cancer expression profiling studies. Clin Cancer Res 9:4811-4818, 2003

48. Ahmed AA, Vias M, lyer NG, et al: Microarray segmentation methods significantly influence data precision. Nucleic Acids Res 32:e50, 2004

49. Ein-Dor L, Kela I, Getz G, et al: Outcome signature genes in breast cancer: Is there a unique set? Bioinformatics 21:171-178, 2005

50. Michiels S, Koscielny S, Hill C: Prediction of cancer outcome with microarrays: A multiple random validation strategy. Lancet 365:488-492, 2005
51. Bair E, Tibshirani R: Semi-supervised methods to predict patient survival from gene expression data. PLoS Biol 2:E108, 2004

52. Kang $Y$, Siegel PM, Shu $W$, et al: A multigenic program mediating breast cancer metastasis to bone. Cancer Cell 3:537-549, 2003

53. Woelfle U, Cloos J, Sauter G, et al: Molecular signature associated with bone marrow micrometastasis in human breast cancer. Cancer Res 63:5679-5684, 2003

54. Minn AJ, Kang $Y$, Serganova I, et al: Distinct organ-specific metastatic potential of individual breast cancer cells and primary tumors. J Clin Invest 115:44-55, 2005

55. Ma XJ, Wang Z, Ryan PD, et al: A twogene expression ratio predicts clinical outcome in breast cancer patients treated with tamoxifen. Cancer Cell 5:607-616, 2004

56. Reid JF, Lusa L, De Cecco L, et al: Limits of predictive models using microarray data for breast cancer clinical treatment outcome. J Natl Cancer Inst 97:927-930, 2005

57. Simon R: Development and validation of therapeutically relevant multi-gene biomarker classifiers. J Natl Cancer Inst 97:866-867, 2005

58. Eden P, Ritz C, Rose $C$, et al: Good old clinical markers have similar power in breast cancer prognosis as microarray gene expression profilers. Eur J Cancer 40:1837-1841, 2004

59. Kattan MW: Evaluating a new marker's predictive contribution. Clin Cancer Res 10:822824, 2004

60. Ayers M, Symmans WF, Stec J, et al: Gene expression profiles predict complete pathologic response to neoadjuvant paclitaxel and fluorouracil, doxorubicin, and cyclophosphamide chemotherapy in breast cancer. J Clin Oncol 22:2284-2293, 2004

61. Iwao-Koizumi K, Matoba R, Ueno N, et al: Prediction of docetaxel response in human breast cancer by gene expression profiling. $\mathrm{J}$ Clin Oncol 23:422-431, 2005

62. Chang JC, Wooten EC, Tsimelzon A, et al: Patterns of resistance and incomplete response to docetaxel by gene expression profiling in breast cancer patients. J Clin Oncol 23:11691177, 2005

63. Burstein HJ, Winer EP: HER2 or not HER2: That is the question. J Clin Oncol 23:3656-3659, 2005

64. Sargent DJ, Conley BA, Allegra C, et al: Clinical trial designs for predictive marker validation in cancer treatment trials. J Clin Oncol 23: 2020-2027, 2005

65. Olivotto IA, Bajdik CD, Ravdin PM, et al: Population-based validation of the prognostic model ADJUVANT! for early breast cancer. J Clin Oncol 23:2716-2725, 2005

66. McGuire WL: Breast cancer prognostic factors: Evaluation guidelines. J Natl Cancer Inst 83:154-155, 1991 